\title{
On the Trail of Paediatric Liver Transplant in South Africa - Social Challenges to Equitable Distribution in Organ Transplantation
}

\author{
TINA C. SIDERIS, Wits Donald Gordon Medical Centre, Johannesburg, South Africa \\ JUNE FABIAN, Wits Donald Gordon Medical Centre, Johannesburg, South Africa and \\ Department of Internal Medicine, Faculty of Health Sciences, University of Witwatersrand, \\ Johannesburg, South Africa*
}

\begin{abstract}
This paper tracks the journey of a family from a remote rural area in South Africa - a 2 year old child born with a life threatening liver disease, and his unemployed mother - who, by a series of contingencies, are sent on the trail of organ transplantation to land at the door of a private organ transplant centre. This case brings into focus the dilemmas that social factors present for equitable distribution of organ transplantation. The paper focuses on two interconnected issues: the link between socio-economic status and access to treatment, and existing practices of rationing. The uncritical conjunction of socio-economic status and organ transplantation disadvantages vulnerable sectors of the population. Yet, social circumstances impact the management of specialized medical treatment, which in itself imposes burdens on those with limited resources. Similarly, although this paper poses questions about indiscriminate practices, it accepts the inevitable rationing of health care.
\end{abstract}

Keywords: organ transplant; paediatric liver transplant; social criteria; rationing; rural health; politics of life; social justice; care

*The authors wish to thank Sister Kim Crymble for her invaluable contribution and support. 


\section{Introduction}

This paper tracks the journey of a family from a remote rural area in South Africa - a 2 year old child born with a life threatening liver disease and his unemployed mother - who, by a series of contingencies, are sent on the trail of organ transplantation to land at the door of a private organ transplant centre. Organ transplantation is widely accepted as standard medical treatment for people suffering from end-stage organ failure. In South Africa, the National Health Act regulates the use of solid organs thereby providing legal parameters for this form of treatment (National Health Act, 2003, chapter 8). The case study that motivated this paper brings into focus the dilemmas that social factors present for the equitable distribution of organ transplantation. Two interconnected issues constitute the focus of this paper: the link between socio-economic status and access to treatment, and existing practices of rationing. The paper questions the uncritical conjunction of socio-economic status and organ transplantation. Yet, by acknowledging the role that social circumstances play in the management of complex medical treatment as well as the burden specialized treatment imposes on those with limited resources, no pretense to easy solutions is made. Similarly, though noting the inevitability of rationing, the paper poses critical questions about indiscriminate practices.

A brief socio-historical overview of transplantation in South Africa illustrates why and how social criteria have been implicated. This discussion is followed by a consideration of the distribution and rationing of treatment across state and private health sectors in South Africa, using paediatric liver transplantation as an illustration. The principle of nonabandonment put forward by medical ethicists who argue for extension of life-saving treatment to children by the state health sector and proposals for a nationally funded paediatric liver transplant centre in South Africa are discussed in the next section of the paper. A more detailed description of the social background of the child on whom this paper 
focuses and the implications of his social circumstances for making use of specialized medical treatment are then provided. Finally, the paper reflects on how deliberation on such cases can generate critical thinking.

The first solid organ transplanted in South Africa was a kidney from a living donor, taking place on $25^{\text {th }}$ August 1966. The surgical team invited a pioneer of transplantation from the USA, Thomas Starzl, to perform the first transplant in Johannesburg (Starzl, 1992). The 33 year old recipient died a month later, having lost the kidney due to rejection. Shortly thereafter, the first heart transplant in the world was performed by Christiaan Barnard at Groote Schuur Hospital in Cape Town in1967. The recipient died 18 days after surgery as a result of sepsis. While kidney transplantation grew in Johannesburg, adult liver transplantation was abandoned due to high fatalities. Similarly, paediatric liver transplantation established at Cape Town's Red Cross War Memorial Children's Hospital (RCWMCH) in 1987 was suspended due to the death of first child patient. In 1991, the programme was restarted and remains active (Spearman et al., 2006). With advances in compatibility testing for donors and recipients, better immunosuppressive treatment, and a growth in the pool of professional experience, patient and graft survival improved (Myburgh et al., 1983), allowing solid organ transplantation to transition from experimental to acceptable medical practice with the expertise, initially, located primarily within the state sector.

Currently in South Africa, the provision of organ transplantation is complicated by social realities that shape population health, health care needs, and access to health care. South Africa is battling an epidemic of infectious diseases in the form of HIV and TB, and a rise in non-communicable diseases that eclipses the rates in developed countries by two to three times, affecting predominantly rural and poor urban communities (Mayosi et al., 2009). 
These health care needs are superimposed on a population with high rates of maternal and infant mortality and high rates of injury and violence.

Organ transplants involve significant financial costs constituted by the need to secure and train health care professionals with highly specialized skills, appropriately equipped health care facilities, the provision of life-long immunosuppression, regular follow-up and support of recipients, and the considerable costs of organ procurement. Paediatric organ transplantation has consequences for children and their families that extend beyond the procedure itself. These include psycho-social and financial implications that can be exacerbated where one parent is the living donor, a choice that is becoming more common. In well-resourced transplant centres, required support may be more accessible. Regrettably, in South Africa, this is not realistic with significant social and material costs to those whose socio-economic circumstances already put them under pressure.

In health care systems that prioritize primary and secondary health care, such as South Africa, the provision of high-cost, specialized medical treatment provokes critical questions about equitable access and balancing distribution equitably across private and state sectors (Millar \& Hamza 2012; Spearman \& McCulloch 2014). Noting the complexity of reconciling provision of high cost biomedical treatment in a context where primary and secondary health care demands are pressing, paediatricians and surgeons involved in treating liver disease in children make the point that while liver transplantation is high-cost, it is a relatively lowdemand technology (Lala et.al., 2014).

In addition to these wider socio-economic considerations, organ transplantation brings socio-economic realities to the fore in more particular ways. The transfer of organs from one body to another contests death and simultaneously provides the impetus for the redefinition of death in order to procure organs viable for transplant (Lock, 2002). Hence, body parts 
assume value. Their exchange has implicated some organ transplantation centres in economic arrangements, always complex, sometimes sinister. Medical anthropologists and sociologists have registered concerns about the commodification of the body, organ trafficking, and inequities in transplant medicine (Fox \& Swazey, 1992; Scheper-Hughes, 2004; ScheperHughes, 2000).

Over and above the supply and demand of bodily matter, the biological requirements consequent to the co-existence of distinct DNA in one body have drawn biological and socioeconomic matters together. More specifically, because immune suppressed patients require vigilance against infection, a view has prevailed about living conditions that provide amenities for optimal hygiene as pre-requisites for viable organ transplantation. Thus, the biological requirements of organ transplantation implicate socio-economic realities.

Although the South African Department of Health has legislated use of solid organs for organ donation (National Health Act, 2003, chapter 8), not all affected individuals are automatically eligible, nor is every organ transplant equally accessible. The kidney is the most widely transplanted organ, far in excess of other solid organs, including the liver. Thus far, there are 18 hospitals participating in transplantation in South Africa with these facilities located in only 4 of the 9 provinces. Eight of these hospitals are state hospitals and all perform kidney transplantation, however Johannesburg and Cape Town state hospitals are the only two that offer liver transplantation.

In August 2004, a liver transplant programme started at Wits Donald Gordon Medical Centre (WDGMC), a private medical facility. An agreement between Charlotte Maxeke Johannesburg Academic Hospital (CMJAH), a state hospital, and WDGMC allows state patients to receive liver transplants at costs subsidized by WDGMC. Funded and state patients are listed on separate waiting lists. A quota system is in place whereby for a fixed 
number of privately funded patients transplanted, one state patient is transplanted. This does not apply to patients in acute fulminant liver failure, all of whom receive equal access. At present no state hospital provides heart and lung transplantation for Gauteng and its referral sites.

Following the establishment of the liver transplant programme at WDGMC in 2004, a paediatric liver transplant was performed in November 2005. Between November 2005 and December 2011, a total of 29 children received liver transplants. In addition to the shortage of donor organs, the professionals involved identified lack of resident expertise in paediatric hepatology and paediatric intensive care as the reasons for the "stagnation" of the programme. In 2012 a paediatric hepatologist, a paediatric intensivist, and a liver transplant surgeon with expertise in living donor liver transplantation joined the existing team. Since then the programme has expanded rapidly performing another 30 transplants by 2014 , including 12 living donor transplants (Loveland et al., 2014).

Criteria for transplantation include a focus on patient survival of the procedure, and potential benefit from the transplant for a relatively long period. Because immunosuppression places patients at risk of life-threatening infection and recipients should have ready access to specialized care, many clinicians feel that social conditions have a bearing on outcome. Without empirical evidence these views come across as common sense. There are varying essential pre-conditions for transplantation, including running water in the home, internal sanitation facilities, being able to have a meal before taking medication, and having available transport to access health care swiftly in case of infection. To date there are no published South African studies that explicate the impact of social circumstances on graft survival and recipient mortality. Hence, the uncritical application of social circumstances to selection could result in indiscriminate rationing that disadvantages particular sectors of the population. 


\section{Rationing - Access to medical treatment and the "inequality of lives"}

In the field of health care, rationing, an economic term, refers to the allocation of health care goods and service either according to ability to pay or by availability of goods, or indeed, by 'penalising' for pre-existing physical conditions. Debates in medical ethics take as the starting point the inevitability of rationing or limit setting and hence focus on what mechanisms could ensure fair and just allocation. Some propose broad, inclusive, and public democratic deliberation as the only mechanism to ensure just outcomes and suggest public access to the decision making process (Fleck, 1994). The above form of deliberative democracy is not without critics who point to an impossible tension in health care: “...our inclination to meet people's needs pushes us toward an unsustainable policy, just as our inclination to think we need limits makes us realize we have no simple answers about how to set them" (Daniels, 2010).

In a commentary on humanitarian work, medical anthropologist Fassin $(2009 ; 2007)$, registers a 'politics of life' whereby he argues that social inequalities inform the evaluation of which lives are to be saved and which to be risked. When medical review boards or transplant panels deliberate on who qualifies for treatment, anatomical, physiological, biochemical, and surgical considerations guided by bioethical principles determine the listing of candidates for transplant and the allocation of organs. Yet, as has been suggested above, where there is an uncritical coupling of biological and social economic status, the frameworks of autonomous decision-making, informed consent, beneficence, and maximizing utility can obscure socio-political limitations to choice and possibility.

In other words, considering the concrete conditions in which normative frameworks are applied and enacted reveals constraints and possibilities. For example, the lack of resources (including finances, transport, and knowledge) with which to make use of health 
care limits the meaningful choice that liberty and agency imply in bioethical conceptions of autonomy. Some make the point more strongly: "Autonomy-based bioethics has a tendency to distort the relationship between individuals and the world. On the one hand, it exaggerates the power and range of individual agency; furthermore, it underestimates the impact of society, culture and environment, both on individual decision-making and on health" (Azetsop \& Rennie, 2010, 3).

In South Africa there is a paucity of systematic research on health care rationing. In the field of treatment for end-stage organ failure, the distribution of renal replacement therapy is better researched relative to treatment for diseases and treatment of other solid organ failure. Hence, in order to explore the practices of rationing in this field of organ transplantation, the following section focuses on access to renal replacement therapy dialysis and kidney transplant.

In South Africa, a dichotomy exists in policy regulating access to renal replacement therapy. In the private sector, funders are obliged to offer renal replacement to all their members who present with end-stage disease. However, in the state sector, only those proven eligible for kidney transplantation are considered for chronic dialysis. However, even this does not guarantee receipt of treatment. Paradoxically, this policy compromises the large majority of the population who cannot afford private health care. Some argue that those dependent on state health care are further disadvantaged by “inefficiencies, incompetent management, corruption and lack of accountability" which compromise scare resources (Dhai, 2012, 2).

By investing in chronic dialysis units, the South African Department of Health has created a need for scarce resources and an expectation of treatment (Scheper-Hughes, 2000). In an article addressing the selection of patients for treatment, Professor Moosa of Tygerberg 
Academic Hospital, a state facility, pointed out that $52 \%$ of patients that require dialysis are turned away (Moosa \& Kidd, 2006). In reality this means that these individuals are turned away to die. It is important to note that this form of rationing is determined by government allocation of resources. The South African Renal Registry Annual Report makes this clearer (Davids et al., 2013).

Since 1994, rates of treatment for end-stage renal disease (ESRD)/per million population (pmp) improved from 70 (1994) to 167 (2013). Closer scrutiny of the data reveals significant disparities in the distribution of treatment across state and private sectors. The treatment rate in 2013 for state sector patients is essentially unchanged at $71 \mathrm{pmp}$ (3150 patients) as compared to 648 pmp (5690 patients) in the private sector. Between 1994 and 2013 state sector treatment centres offering dialysis increased from 26 to 29. Private sector facilities increased from 5 to 178 , clearly showing that increased access to treatment is confined to the private sector, whose rates are comparable to those achieved in other middle income countries (White et al., 2008).

In state facilities, because access to this form of care is severely limited, the application of the criterion "eligibility for transplantation" is in essence a form of rationing; however, there are no national guidelines for rationing. The responsibility for deciding which particular patient to 'make live' and which to 'reject into death' is deferred to clinicians for whom the burden is overwhelming (Fassin 2009, 54; Moosa \& Kidd, 2006). Rationing is inevitable when it comes to the public provision of goods and services. As Hoggett (2006b) points out in his work on public service organizations in the UK, the demand for free public services is potentially unlimited. Thus, public service professionals are called to make rationing decisions according to the policy of the day. While there is no doubt that public 'servants' must use their discretion, it is reasonable to argue that policies are needed to provide the boundaries within which judgements are applied. Moosa and Kidd (2006) frankly 
note that in their experience socio-economic factors influenced the decision to accept patients for renal replacement therapy more significantly than did medical factors. Race, gender, age, employment status, and proximity to a treatment centre are factors that influenced access to renal replacement therapy. Similar research and documentation on the selection of patients for liver transplantation is not available.

That socio-economic factors are key to accessing to organ transplantation is not unique to South Africa. International literature reveals that social criteria play a significant role in selection for transplantation. A retrospective analysis of 749 patients with hepatocellular carcinoma referred to the only liver transplant centre in the State of Hawaii found that fewer Pacific Islanders underwent transplants than Whites and Asians; transplant patients were younger than those not transplanted, were male, had completed high school education, and had private medical insurance. Patients with no identified employment were less likely to receive transplants (Wong et al., 2012).

Furthermore, the literature notes that costs of immune suppressant medication for patients without medical insurance or with limited coverage constitute a significant impediment to successful organ transplant (Simmerling, 2007). Costs of the procedure excluded a similar category of people in Iran, where Lankarani et al. (2012) report a closing of the gap between high and low socio-economic class access to liver transplantation since the Ministry of Health decided to cover costs.

Apart from real financial obstacles to sustaining treatment post-transplant, the literature does not provide systematic evidence about the impact of social factors on outcomes. In South Africa, the use of access to social amenities and resources for rationing organ transplantation is not consistent across centres, nor, as noted above, is the use of these criteria based on empirical evidence. In South Africa, equitable distribution of such care 
requires research on i) the conjunction of social conditions and decision making about who to treat that exists at some transplant centre; ii) patient and graft survival in patients whose living conditions challenge these specifications; and iii) evidence based guidelines to assist clinicians in the inevitable process of rationing.

\section{Non-abandonment}

In support of greater equity, some have proposed a policy commitment to nonabandonment thereby explicitly challenging financial resources as the criterion for access to treatment (Landman \& Henley, 1999). A patient is abandoned when a physician stops treatment before the patient is recovered or the contractual relationship has ended and/or if a physician ceases treatment without referral to another practitioner (McQuoid-Mason, 2011).

Mindful of extreme health needs in post-apartheid South Africa, the scarcity of resources, the state's emphasis on prevention and primary care rather than tertiary care, and the commitment to health and equality in the Bill of Rights, Landman and Henley $(1999,225)$ propose that non-abandonment provides a rational moral framework for decision making on specialized medical treatment of children. By non-abandonment they suggest that "no identified area of vital health care need should be excluded"(Landman and Henley, 1999, 225). Unless specialized treatment is provided in the state sector, they argue, payment becomes the criterion for children to access life-saving or life-enhancing treatment.

More recent proposals addressing paediatric organ transplant similarly suggest a nationally funded programme to be implemented by national health authorities (Loveland et al., 2014). These authors suggest that for children without private medical insurance it is feasible to conduct pre-transplant workup at state facilities for children with constant monitoring of the clinical and cost effectiveness of protocols (Lala et al., 2014, 832; Loveland et al., 2014). They argue that the current paediatric transplant centres at WDGMC 
in Johannesburg and the Red Cross War Memorial Children's Hospital (RCWMH) in Cape Town are well established and should not be duplicated. Post-transplant care, they propose, should be available at regional and academic state facilities funded by the national funding programme to provide life-long care post-transplant for children who rely on state health care (Loveland et al., 2014). With reference to the large state hospital in Soweto, the Chris Hani Baragwanath Academic Hospital (CHBAH), they point out that increasing numbers of children are being referred to the paediatric liver transplant centre at WDGMC.

These proposals, which draw on an ethics of fairness and justice, are appealing and go a long way towards addressing inequities in access to specialized medical treatment. Nevertheless, they do not address the questions raised in this paper regarding the use of social conditions, in particular living conditions and basic amenities, as selection criteria for organ transplantation. The case study that stimulated the writing of this paper provides a more complex picture of the dilemmas presented by social condition. In this case, socio-economic status and the conditions of life in the particular social location in which this child lives reveal the challenges posed to providing and using specialized medical treatment such as organ transplant and provoke questions about just and fair distribution of health over and above the hard choices that rationing requires and how fair decisions about such limits are made (Daniels, 2010). Poor road and service infrastructure, limited health care, unemployment, and illiteracy place considerable constraints on being able to sustain the kind of long term treatment and lifestyle that organ transplantation requires. Thus, could it be that in the case of organ transplantation such structural conditions pose significant limitations on just distribution?

\section{On the Trail of Organ Transplantation}


Baby S was born in the Eastern Cape in a rural district called Mqanduli. At 24 months he had been in and out of hospitals diagnosed with a life threatening liver disease whose symptoms, usually presenting within a few weeks of birth, include jaundice, unrelenting itchiness, malabsorption resulting in growth retardation, and eventually liver failure and death. Liver transplantation is currently considered the only option for life extension.

The district of Mqanduli, which was part of a 'homeland' area under the apartheid Bantustan policy, is made up of a cluster of 44 villages. This is amongst the poorest areas of the country with high rates of unemployment (44\%), high rates of illiteracy, and minimal infrastructure. According to a 2007 Department of Water Affairs report only $19.6 \%$ of household have piped water in the home; $14.1 \%$ have yard taps, and $47 \%$ of households have access to taps within 200m from their stands (Umvoto Africa, 2011).

The Health System's Trust District Health Barometer (Massyn et al., 2013) suggests extreme health care needs some of which are directly related to poverty - the highest diarrhoea fatality rate for children under 5 in the province and in the country; the fourth highest pneumonia fatality rate for children under 5 in the country; and a severe acute malnutrition incidence of 4.5 per 1000 children under 5. Primary health care clinics servicing Mqanduli vary between a distance of 2 and $20 \mathrm{~km}$ from individual households. These clinics refer to Zithulele hospital, which was established in 1956 by Christian Missionaries. Handed over to the Department of Health, the hospital, staffed by a multi- disciplinary team of 22 professionals, allied health and support staff, provides non-emergency hospital services to surrounding villages with a population of roughly 130000 people (Gaunt, 2010). Maternal and child health care and HIV treatment are a key focus of health care.

The impoverished social conditions that prevail in the Mqanduli sub-district and others like it do not support population health and complicate the use of health care resources. 
That this is a rural area of specific type - a former 'homeland' area - goes some way to explaining how geography, inability to pay, and impoverished social conditions converge. Under apartheid, 'homelands' were sites of reproduction of labour for mines and industries in the distant industrial centres, and they served as dumping grounds for surplus labour. Notoriously underdeveloped with little or no infrastructure, subsistence farming on environmentally denuded land, many of these areas remain distant from South African economic centres (Jensen, 2007). Research suggests that prospects for economic growth remain bleak, given the remoteness from industrial centres, failure to develop industrial bases, and underfunding of services and infrastructure (Nhlapo, Kasumba \&Ruhiiga, 2011). They remain areas of high unemployment with poor infrastructure and limited services, as is well illustrated by the household of S and his family.

$\mathrm{Z}$, the 20 year old mother of S, comes from a household typical of the area. A report compiled by a local maternal and child health organization describes the two-roomed house in which she lives as a "flat" referring to the flat roof. One room doubles as kitchen and bedroom for mother and child. The other is a bedroom for his grandmother. Their toilet is $100 \mathrm{~m}$ away from the "flat." There is a water tap about $1 \mathrm{~km}$ up the valley. The house has no electricity. It is situated $39 \mathrm{~km}$ from Zithulele Hospital accessed via road, except for the last 2 $\mathrm{km}$ which requires hiking on foot over a valley and crossing a river.

Z's mother is also unemployed. Her father, a migrant worker, has worked in the platinum mines in the notorious Marikana district, for most of his life. The family relies on his salary, supplemented by a child care grant. At time of writing he was among the miners who had been on strike for over 5 months. The 23 year old father of the child remains unemployed. Their child's need for highly specialized and costly medical treatment stands out against this background of poverty and scarcity of health resources. 
Z's journey of over $800 \mathrm{~km}$ on the trail of treatment for her son from Mqanduli to Chris Hani Baragwanath Academic Hospital (CHBAH) in Soweto, Johannesburg, speaks of determination to access treatment. Her unplanned pregnancy and normal vaginal delivery were uneventful, but at 4 weeks when his yellow eyes had not cleared, she took him back to the local clinic who advised sunlight, to no effect. By 5 months, disturbed by the baby's distended abdomen, the clinic referred to Zithulele hospital and from there, on to a tertiary care hospital in the city of East London, some $200 \mathrm{~km}$ from her home. A biopsy of his liver was taken after which no clear directive was obtained.

Increasingly desperate, Z's mother, S's grandmother, took him to Rustenburg, the district where her husband works in the platinum mines. Located in the North West province, Rustenburg is a distance of some $980 \mathrm{~km}$ from their home in the Eastern Cape. Making this 11 hour journey by public transplant is long and tiring by most people's standards. Made with a sick child, the journey was arduous. It was from the local clinic in Rustenburg that they were referred another $120 \mathrm{~km}$ to $\mathrm{CHBAH}$ in Gauteng. There they were informed of the severity of his condition and that he would require his liver to be replaced. The family returned to Mqanduli, where he deteriorated with extreme swelling of his abdomen. A doctor at Zithulele advised $\mathrm{Z}$ to take her baby back to $\mathrm{CHBAH}$ urgently and covered the costs of their return by air flight. Following several weeks admission at CHBAH, baby S was brought to the attention of the liver transplant team at WDGMC.

The unique personal characteristics and internal psychic world of this young mother that might help to explain her determination have not been explored. Neither have the personal moral conflicts that she may have faced been discussed. When she was told about strenuous post-transplant treatment and lifestyle requirement, tears welled up. She reflected aloud that many women of her age and circumstances would have abandoned a child this sick. She may be right, but hers is not an isolated petition. Increasingly, in post-apartheid 
South Africa citizens are informed about health care options and are demanding access. In other words, the poor can and do make claims on the state. Even more specifically the sick can and do make claims instantiating biological citizenship, such that politics are clearly implicated in access to health care (Fassin, 2009; Marsland \& Prince, 2012).

Although it is within realistic possibility to maintain requisite hygiene without internal tapped water, sanitation, and electricity, the challenges are amplified. That the house of our young protagonist is far from the local hospital, further from tertiary care, and, due to the local political geography, not accessible by road renders him more precarious in a medical emergency. His milk teeth are decayed, a mark not of his disease, but of the limitations of his nutrition, which is unlikely to improve dramatically given the restricted socio-economic prospects of his family. Transport to and from the clinic and local hospital will place an additional strain on the resources of this family.

Noting the spread of science, biomedicine, and technology within the context of inequality, Marsland and Prince (2012) ask at what price these technologies are accessed. Of course, one could ask what will be the social and personal costs to baby $\mathrm{S}$ and his family for receiving organ transplantation. In this regard it is worth reflecting on research from the World Nephrology Congress of Nephrology 2015 held in Cape Town, South Africa. Staff from Paediatric Nephrology at CMJAH interviewed caregivers to evaluate the economic burden of chronic dialysis on families of 19 paediatric patients. The mean monthly family income was R2946.00 per month, the equivalent of USD 245.00. On this monthly income, in a family of four, the monthly amount available to each member of the family is R737.00 (R25.00 per day, equivalent to USD 2.00 per day). For children on haemodialysis, families spend $27.1 \%$ of the family income on transport thereby reducing the monthly amount available per individual family member to R537.00 (R18.00, equivalent to USD 1.50 per day) (Levy et al., 2015). Clearly, social inequalities not only impact on the capacity to use 
specialized medical treatment, but also significantly affect the experience of sustaining lifeenhancing treatment.

Of course when faced with an individual child whose life can be saved, these balances are difficult if not impossible to calculate. This is even more so for families, as it happened with Z. When offered the possibility of treatment, how can a mother say no to her son, to herself? Herein lies the dilemma.

\section{Care and Justice}

The liver transplant panel at the private medical institution to which baby S presented considered his social conditions within a logic of constraints and possibilities, rather than exclusion. Access to a health care facility for clinical examination and other investigations, such as drawing and testing blood and access to immune suppression medicine after surgery were the more important considerations. The role played by Zithulele Hospital and its professionals was the critical deciding factor in listing him for treatment. In light of the legacy of the apartheid homeland system, Zithulele challenges conventional wisdom about public rural health facilities and the possibilities of rural health care.

Public service organizations are constantly confronted with competing claims: care versus justice and how to balance the care needs of the individual with the needs of the group (Hoggett, 2006b). Baby S confronted Zithulele hospital with exactly this dilemma. Communication with hospital staff reveals that without clear policy guidelines, they were called upon to apply discretion. Judgements were made not simply by management or professional staff, but included consultation with citizens in civic organizations, local government, traditional authorities, and provincial health authorities to imagine making the medical management of a liver transplant patient in a remote rural village possible. These stakeholders and agencies made practical arrangements to facilitate successful treatment after 
transplantation. In this way Zithulele sought to re-estimate the value assigned to a particular rural life.

What do we make of the decision taken by the professionals on the transplant panel? The fact that baby $\mathrm{S}$ was listed for transplantation constitutes non- abandonment. If compassion involves a motivation to alleviate the suffering of another then compassion very evidently played a role. Could it be that compassion, and the principle of non-abandonment as a deontological rule, moved these professionals in a private institution to compromise on payment of their highly skilled service?

Some would argue that the case of Baby S should engender a more socially critical view, whereby compassion for suffering of the other must be fused with anger at the injustices that result in social suffering (Hoggett, 2006a, 161). In this way the ethics of care, in this instance care for individual patients, might be fused with a capacity to 'think' critically about social injustice or the unfair distributions of burdens, perhaps resulting in a capacity to question the "inequality of lives."

\section{Conclusion}

There is a danger of constructing the family of S and others like them as heroically resilient, confirming a view that assigns the health and the use of health care to individual choice, or self-responsibility. As has been noted, considering concrete conditions in which health care is sought complicates the normative framework of autonomy. Azetsop and Rennie (2010) point out that " $[\mathrm{w}]$ hen people can barely afford the cost of care or satisfy the nutritional requirements for a good recovery, the ethics of medical encounter should be understood differently and expressed in different terms than patient choice" (2). Similarly, there is a danger of confining attention to the suffering and treatment of individual children. Situating baby S, his life threatening condition, and his need for organ transplant in a socio- 
political context registers a set of socially situated dilemmas. On the one hand, this paper cautions that the uncritical application of social conditions as criteria for treatment bears the danger of discriminating against the impoverished and marginalised. On the other hand the paper suggests that the burden of using specialized medical treatment is loaded by conditions of poverty. Thus, baby S and his need for medical treatment evoke Fassin's (2007) 'politics of life.'

Equally, there is a danger of idealising the work of Zithulele Hospital, thereby diverting attention from the purpose of public service organizations and the moral ambivalences they must confront, not least the tension between care for the individual and mindfulness of social justice (Hogget, 2006a). In this regard it is significant that $\mathrm{S}$ and his grandfather are both involved in a struggle for access to resources. When this grandfather retires from mining and returns to his village he will in all likelihood be suffering from a common occupational hazard - debilitating, terminal lung disease - for which there is no treatment centre in the Eastern Cape. Despite the fact that a large majority of miners are drawn from this area, neither the mining companies nor the state have made provision for treatment or rehabilitation for miner's diseases. Nor indeed has the state compelled mining corporations to provide health care. It is not hard to note the unfair distribution of burdens, neither is it hard to juxtapose the cost of organ transplantation for a few, against the cost of providing rehabilitation facilities to the many. Yet, as has been noted above, when faced with an individual child whose life can be saved these balances are difficult to calculate.

Tracking baby $\mathrm{S}$ on the trail of organ transplant as it has traversed urban and rural, public and private sectors reveals the dilemmas that claims to specialized medical treatment give rise to in contexts of social inequality, particularly where structural conditions limit individual choice and the just distribution of specific treatments. While this paper registers social inequalities in the access to and use of specialized treatment that are linked to factors 
beyond the decisions taken by individual clinicians and review boards, it nevertheless suggests the importance of reflecting on the social values and priorities underlying decisionmaking about claims to treatment. Health care rationing may be inevitable. That the indiscriminate use of social conditions as selection criteria inevitably disadvantages the poorest sectors of the population of treatment considered standard, provides food for thought. 


\section{References}

Azetsop, J. \& Rennie, S. (2010). Principlism, medical individualism, and health promotion in resource-poor countries: can autonomy-based bioethics promote social justice and population health? Philosophy, Ethics and Humanities in Medicine, 5 (1), 1-10.

Daniels, N. (2010). Just Caring: Health care rationing and democratic deliberation, by L. Fleck. Notre Dame Philosophical Reviews. Retrieved from: https://ndpr.nd.edu/news/24413-just-caring-health-care-rationing-and-democraticdeliberation

Davids M.R., Marais N., Balbir Singh G.K. \& Jacobs J.C. (2013). South African Renal Registry Annual Report 2013. Cape Town, South Africa. Retrieved from: www.sarenalsociety.org/Registry/2013/SA-RenalRegistry_2013.pdf

Fassin, D. (2007). Humanitarianism as a politics of life. Public Culture, 19(3), 499.

Fassin, D. (2009). Another politics of life is possible. Theory, Culture \& Society, 26(5), 4460.

Fleck, L.M. (1994). Just caring: Oregon, health care rationing, and informed democratic deliberation. Journal of Medicine and Philosophy, 19(4), 367-388.

Fleck. L.M. (2009). Just caring: health care rationing and democratic deliberation. Oxford University Press, New York, USA.

Fox, R.C. \& Swazey, J.P. (1992). Spare parts: Organ replacement in American society. Oxford University Press, New York, USA.

Gaunt, C. B. (2010). Are we winning? Improving perinatal outcomes at a deeply rural district hospital in South Africa. South African Medical Journal, 100(2), 101- 104. 
Hoggett, P. (2006a). Pity, compassion, solidarity. In Clarke, S., Hoggett, P. \& Thompson, E. (Eds). Emotion, politics and society (145-161). Palgrave Macmillan: Basingstoke, United Kingdom.

Hoggett, P. (2006b). Conflict, ambivalence, and the contested purpose of public organizations. Human Relations, 59(2), 175-194.

Jensen, S. B. (2007). Through the Lens of Crime: Land claims and contestations of citizenship on the frontier of the South African state. In L. Buur, S. Jensen, \& F. Stepputat (Eds.), The Security-Development Nexus: Expressions of sovereignty and securitization in Southern Africa (193-211). Nordiska Afrikainstitutet: Uppsala, Sweden.

Lala, S.G., Britz, R., Botha, J. \& Loveland, J. (2014). Paediatric liver transplantation for children treated at public health facilities in South Africa: Time for change: Paediatric hepatobiliary-opinion. South African Medical Journal, 104(11), 829-832.

Landman, W. A. \& Henley, L.D. (1999). Equitable rationing of highly specialised health care services for children: A perspective from South Africa. Journal of Medical Ethics, 25(3), 224-229.

Lankarani, K.B., Mahmoodi, M., Gholami, S., Mehravar, S., Malekhosseini, S.A., Heydari S.T., Zarei, E., Salahi, H., Nikeghbalian, S. \&Taghavi, S.A. (2012). Reducing social disparity in liver transplantation utilization through governmental financial support. Hepatitis Monthly, 12(11) e6463.

Levy, C.S., Sangweni, B., Bello, A., Obiagwu, P., Mudi, A., Moonsamy, G. \& Khumalo, T. (2015). Financial costs to families of children on long term renal replacement therapy 
(RRT) at a tertiary health care centre in Johannesburg, South Africa. ISN World Congress of Nephrology, 13-17 March, Cape Town, South Africa.

Lock, M.M. (2002). Twice dead: Organ Transplants and the Reinvention of Death. University of California Press: California, USA.

Loveland, J., Britz, R., Joseph, C., Sparaco A,. Zuckerman, M., Langnas, A., Schleicher, G., Strobele, B., Moshesh, P. \& Botha, J. (2014). Paediatric liver transplantation in Johannesburg revisited: 59 transplants and challenges met: paediatric hepatobiliaryresearch. South African Medical Journal, 104(11), 799-802.

Marsland, R. \& Prince R. (2012). What is life worth? Exploring biomedical interventions, survival, and the politics of life. Medical Anthropology Quarterly, 26(4), 453-469.

Massyn, N., Day C., Dombo M., Barron, P., English, R. \& Padarath, A. (2013). Section B: Profile Eastern Cape Province. District Heath Barometer 2012/2013. Health Systems Trust. Durban, South Africa. Retrieved from: www.hst.org.za/publications/districthealth-barometer-201213

Mayosi, B.M., Flisher, A.J., Lalloo, U.G., Sitas, F., Tollman, S.M. \& Bradshaw, D. (2009). The burden of non-communicable diseases in South Africa. The Lancet, 374(9693), 934-947.

McQuoid-Mason, D. (2011). Medical ethics and the payment of fees before treatment. South African Medical Journal, 101(11), 798-799.

Mgaquelwa, A. (October 11, 2013). Girl, 3, 'too poor to live', Dispatch Live (Online). Retrieved from: http://www.dispatchlive.co.za/news/14790/ 
Millar, A. J.W. \& Hamza, A.F. (2012). Liver transplantation in an African setting. Seminars in Pediatric Surgery, 21(2), 164-171.

Moosa, M. \& Kidd, M. (2006). The dangers of rationing dialysis treatment: the dilemma facing a developing country. Kidney International, 70(6), 1107-1114.

Myburgh, J., Botha, J., Meyers, A., Smit, J., Milne, F.J., Thomson, P., Beale, P. \& Seggie, J. (1983). The treatment of end-stage renal disease at the Johannesburg Hospital: a 17year experience. Part II. The role of transplantation. South African Medical Journal, 64(14), 522-527.

National Health Act, No 61 (2003). Department of Health, South Africa. Retrieved from: www.chr.up.ac.za/undp/domestic/docs/legislation_55.pdf

Nhlapo, M., Kasumba, H., \& Ruhiiga, T. (2011). Growth challenges of homeland towns in post-apartheid South Africa. Journal of Social Sciences, 29(1), 47-56.

Scheper-Hughes, N. (2004). Parts unknown. Undercover ethnography of the organstrafficking underworld. Ethnography, 5(1), 29-73.

Scheper-Hughes, N. (2000). The global traffic in human organs. Current Anthropology, 41(2), 191-224.

Simmerling, M. (2007). Beyond Scarcity: Poverty as a contraindication for organ transplantation. Virtual Mentor, 9(6), 441-445.

Spearman, C.W.N. Paediatric Liver Transplantation (Powerpoint Presentation Online). Department of Paediatrics, University of Cape Town. Retrieved from: http://www.paediatrics.uct.ac.za/sites/default/files/image_tool/images/38/Paediatric\%20 Liver\%20Transplantation\%20.pdf 
Spearman, C. W.N \& McCulloch, M. (2014). Challenges for paediatric transplantation in Africa. Pediatric Transplantation, 18(7), 668-674.

Spearman, C.W.N., McCulloch M., Millar A.J.W., Burger, H., Numanoglu, A., Goddard E., Gajjar, P., Davies, C., Muller, E. \& McCurdie, F. (2006). Liver transplantation at Red Cross War Memorial Children's Hospital. South African Medical Journal, 96(9), 960963.

Starzl, T. E. (1992). The puzzle people: Memoirs of a transplant surgeon, University of Pittsburgh Press: Pittsburgh, USA.

Tronto, J. C. (2013). Caring democracy: Markets, equality, and justice. New York University Press: New York, USA.

Umvoto Africa (Pty) Ltd. (2011). Reconciliation Strategy for Mqanduli, Hole in the Wall, Qunu, Viedgesville, Coffee Bay and surrounding villages. Eastern Cape Municipality, South Africa.

White, S.L., Chadban, S.J., Jan, S., Chapman, J. R. \& Cass, A. (2008). How can we achieve global equity in provision of renal replacement therapy? Bulletin World Health Organisation, 86(3), 229-237.

Wong, L. L., Hernandez B., Kwee, S., Albright, CL., Okimoto, G. \& Tsai, N. (2012). Health care disparities in Asians and Pacific Islanders with hepatocellular cancer. American Journal of Surgery, 203(6), 726-732.

\footnotetext{
* POSTSCRIPT With deep regret, it is important to report that our young protagonist, baby $S$, died before organ transplantation. Following vaccination for measles he was temporarily suspended from the transplant list as he would not have been transplantable during the incubation period. His mother took the opportunity to make a visit to her family in the Eastern Cape. Whilst there he contracted an infection and died. The capacity of Zithulele Hospital to manage recovery and treatment of a liver transplant recipient was not tested.
} 